\title{
Cooperative Rendezvous and Docking for Underwater Robots Using Model Predictive Control and Dual Decomposition
}

Nielsen, Mikkel Cornelius; Johansen, Tor Arne; Blanke, Mogens

Published in:

Proceedings of European Control Conference 2018

Link to article, DOI:

10.23919/ECC.2018.8550366

Publication date:

2018

Document Version

Peer reviewed version

Link back to DTU Orbit

Citation (APA):

Nielsen, M. C., Johansen, T. A., \& Blanke, M. (2018). Cooperative Rendezvous and Docking for Underwater Robots Using Model Predictive Control and Dual Decomposition. In Proceedings of European Control Conference 2018 (pp. 14-19). IEEE. https://doi.org/10.23919/ECC.2018.8550366

\section{General rights}

Copyright and moral rights for the publications made accessible in the public portal are retained by the authors and/or other copyright owners and it is a condition of accessing publications that users recognise and abide by the legal requirements associated with these rights.

- Users may download and print one copy of any publication from the public portal for the purpose of private study or research.

- You may not further distribute the material or use it for any profit-making activity or commercial gain

- You may freely distribute the URL identifying the publication in the public portal 


\title{
Cooperative Rendezvous and Docking for Underwater Robots using Model Predictive Control and Dual Decomposition
}

\author{
Mikkel Cornelius Nielsen ${ }^{1,2}$, Tor Arne Johansen ${ }^{1}$, Mogens Blanke ${ }^{1,2}$
}

\begin{abstract}
This paper considers the problem of rendezvous and docking with visual constraints in the context of underwater robots with camera-based navigation. The objective is the convergence of the vehicles to a common point while maintaining visual contact. The proposed solution includes the design of a distributed model predictive controller based on dual decomposition, which allows for optimization in a decentralized fashion. The proposed distributed controller enables rendezvous and docking between vehicles while maintaining visual contact.
\end{abstract}

\section{INTRODUCTION}

Intervention, maintenance, and repair (IMR) operations are an increasingly critical area of application in the offshore industry. Deployment of future sub-sea production facilities is anticipated to occur in deeper and more remote locations. The increased depth require more frequent maintenance, which is already a complicated task at places not directly accessible by humans. The offshore IMR operations are typically carried out by Remotely-Operated-Vehicles (ROVs). The vision of the complete autonomy in the future has increased the interest in Autonomous-UnderwaterVehicles with intervention capabilities (I-AUVs). Multiple recent projects have considered persistent autonomy for intervention-type marine vehicles.

The FP7 project PANDORA investigated the problem of physical intervention between underwater vehicles and underwater panels [16], [20]. The TRITON project investigated docking and manipulation between underwater vehicles and stationary facilities [21], [22].

Operating near exposed offshore structures involves considerable risk [32], [31]. The NextGenIMR project considered the mitigation of operational risk in autonomous interventions such as probability of mission success in case of degradation of the AUV during an active mission combined with shared control with human supervisors [29], [28]. Extending the autonomy to multiple I-AUVs could potentially allow for more flexible and fault-tolerant operations [7], [11]. Multi-vehicle systems with interaction capabilities would allow for smaller vehicles with more specialized tasks such as data-muling, transportation of tools, or collection of test samples [33]. In each case, the vehicles would have to rendezvous to make the

\footnotetext{
${ }^{1}$ Centre for Autonomous Marine Operations and Systems, Department of Engineering Cybernetics, Norwegian University of Science and Technology, Trondheim, Norway

${ }^{2}$ Department of Electrical Engineering, Technical University of Denmark, Lyngby, Denmark

This work was supported by the Research Council of Norway through the Centres of Excellence funding scheme, the AMOS project, grant number 223254.
}

exchange while possibly solving another objective - such as structural monitoring - simultaneously.

Rendezvous and docking are not novel concepts, and multiple authors have considered docking for AUVs. However, the most classic examples are under-actuated torpedo type vehicles [2], [6] and [19], whereas the most common types of IMR vehicles are bulky and fully-actuated [4], [27]. The aeronautical literature has investigated rendezvous and docking for spacecrafts [17], [18], [24], [23], but does not consider damping forces due to the low drag coefficient of the thermospheric layer.

The issue of multi-vehicle rendezvous and docking with multiple objectives, input constraints, and visual constraints pose a challenging problem. Model Predictive Control (MPC) is an advanced control strategy often employed to solve problems where constraints pose a central issue in the objectives. Several authors, [5], [8], [12], have investigated distributed MPCs (DMPCs). Dual decomposition is a frequently applied method, which decomposes a large central optimization problem into smaller sub-problems that can be solved locally.

The decomposition transforms the coupled objective function into decoupled objective functions with coupling constraints. Afterwards, the constraints are relaxed using Lagrangian relaxation and the individual vehicles enforce the coupling constraints by manipulation of the dual variables. Multiple authors, [9], [26], [14], [10], have exploited the dual decomposition technique in various contexts. However, none have considered multi-vehicle docking.

This paper proposes a new multi-vehicle docking control strategy. The strategy utilizes a DMPC by dual decomposition to achieve rendezvous while the vehicles maintain visual contact with each other. The rendezvous problem is solved as a consensus problem solved by introducing a consensus point with a coupled equality constraint between the vehicles. Inspired by, [24] and [23], the visual constraint of the cameras are constructed as inequalities. The constraints are decomposed using dual decomposition and subsequently relaxed by Lagrangian relaxation. The dual variables are then updated by negotiation between neighboring vehicles to thereby enforce the relaxed constraints.

The organization of this paper is as follows: Section II describes the model dynamics used in the paper. Then the overall problem is presented in a centralized fashion in Section III. In Section IV the distributed controller is derived from the centralized problem using dual decomposition. Section $\mathrm{V}$ presents and discusses the results of the simulation. 
Finally, Section VI draws the conclusion along with possible future extensions of the study.

\section{Modeling}

This section starts by an assumption made to reduce problem to a planar case. The dive-plane is stabilized by an active attitude and heave control system. The vehicle considered in this paper is a horizontal plane model of three degrees-offreedom (DOF). Two coordinate frames are considered, first the global navigation frame denoted by $\{n\}$ is approximated by a local earth-fixed North-East plane and secondly bodyfixed frames attached to each of the vehicles such that the frames are aligned with the principle axes. The pose of a vehicle in the global frame is denoted $\boldsymbol{\eta}$ is defined as follows:

$$
\boldsymbol{\eta}=[N, E, \psi]^{T}
$$

where $N$ and $E$ are the position coordinates in the NorthEast frame and $\psi$ is the yaw-angle relative to north. The body-fixed velocity vector is denoted $\nu$ and defined as:

$$
\boldsymbol{\nu}=[u, v, r]^{T}
$$

where $u$ is the surge velocity along the longitudinal $x$-axis, $v$ is the sway velocity along the lateral $y$-axis and $r$ is the rotation velocity around the local downward pointing axis $x \times y$. The transformation matrix $\boldsymbol{J}_{\psi}$ defines the kinematic relationship between body-fixed and global frame velocities as follows:

$$
\dot{\boldsymbol{\eta}}=\overbrace{\left[\begin{array}{ccc}
\cos (\psi) & -\sin (\psi) & 0 \\
\sin (\psi) & \cos (\psi) & 0 \\
0 & 0 & 1
\end{array}\right]}^{\boldsymbol{J}_{\psi}} \boldsymbol{\nu} .
$$

Fig. 1 shows the global coordinate frame with the origin denoted $\mathcal{O}_{n}$ along with the two coordinate frames attached to two vehicles.

\section{A. Kinetics}

Underwater vehicles are affected by hydrodynamic effects. As a consequence a vehicle experiences the effect of hydrodynamic damping and added mass.

$$
M \dot{\nu}+D(\nu) \nu=u,
$$

where $\boldsymbol{M}$ and $\boldsymbol{D}$ are the inertia and damping matrix, respectively, and defined as,

$$
\boldsymbol{M} \triangleq \operatorname{diag}\left(m_{11}, m_{22}, m_{33}\right), \boldsymbol{D} \triangleq \operatorname{diag}\left(d_{11}, d_{22}, d_{33}\right),
$$

and $\boldsymbol{u}$ being the force input in each DOF. The model Eq. (3) and (4) is represented on standard Ordinary-DifferentialEquation (ODE) form by aggregating the pose and bodyvelocity vector as follows:

$$
\boldsymbol{x}=\left[\boldsymbol{\eta}^{T}, \boldsymbol{\nu}^{T}\right]^{T}
$$

The combined state evolution can then be written as follows:

$$
\dot{\boldsymbol{x}}=\boldsymbol{f}(\boldsymbol{x}, \boldsymbol{u})
$$

When considering multiple vehicles in Section III, the vehicles associated with the $i$-th vehicle is assigned an index $i$.

\section{Multi-Vehicle Model Predictive Control for DOCKING}

This section defines the consensus and visual constraint, as well as, the centralized MPC controller. Two objectives, consensus and visual constraints, are defined and combined into a centralized controller.

\section{A. Notation}

Consider a multi-vehicle system comprised of $n$ agents. The agents interact through a network defined by a graphtopology $\mathcal{G}$. Agent $i$ in the network correspond to a vertex $v_{i}$ in the graph, the connection between individual agents are defined by the edge $e_{i j}=v_{i} v_{j}$. Together the set of vertices $\mathcal{V}$ and the set of edges $\mathcal{E}$ constitute a graph $\mathcal{G}$.

$$
\mathcal{G}=\{\mathcal{V}, \mathcal{E}\}
$$

Each agent in the graph has a set of neighbors $\mathcal{N}_{i}$ consisting of all vertices in the graph connected to the agent

$$
\mathcal{N}_{i}(\mathcal{G})=\left\{v_{j} \in \mathcal{V} \mid v_{i} v_{j} \in \mathcal{E} \vee v_{j} v_{i} \in \mathcal{E}\right\}
$$

The graph $\mathcal{G}$ in this paper is un-directed. However, the notational convenience, an arbitrary direction is assigned. Thereby there exist a set of predecessors and successors for each vertex,

$$
\begin{aligned}
& \mathcal{N}_{i}^{+}(\mathcal{G})=\left\{v_{j} \in \mathcal{V} \mid v_{i} v_{j} \in \mathcal{E}\right\}, \\
& \mathcal{N}_{i}^{-}(\mathcal{G})=\left\{v_{j} \in \mathcal{V} \mid v_{j} v_{i} \in \mathcal{E}\right\},
\end{aligned}
$$

such that $\mathcal{N}_{i}=\mathcal{N}_{i}^{+} \cup \mathcal{N}_{i}^{-}$.

\section{B. Line-of-Sight Constraints}

To utilize visual navigation between vehicles, it is necessary to restrict the motion between them such that they never loose esight of each other. Inspired by work in [23], [24], and [25], the line-of-sight (LOS) constraints can be formulated as follows:

$$
\begin{aligned}
& \boldsymbol{n}_{i, j}^{+} \boldsymbol{e}_{j, i} \leq 0, \text { for } \forall v_{i} \in \mathcal{V} \wedge v_{j} \in \mathcal{N}_{i}, \\
& \boldsymbol{n}_{i, j}^{-} \boldsymbol{e}_{j, i} \leq 0, \text { for } \forall v_{i} \in \mathcal{V} \wedge v_{j} \in \mathcal{N}_{i},
\end{aligned}
$$

where the normal vectors $\boldsymbol{n}_{i, j}^{+}$, and $\boldsymbol{n}_{i, j}^{-}$are parameterized in the $i$ th vehicles respective camera rotation. Notice, that the $\boldsymbol{n}$ vectors are parameterized in the states, and thus are timevarying. The $\boldsymbol{e}_{j, i}$ is the relative position between the vehicle in the global frame and is assumed measured by the local camera.

$$
\boldsymbol{e}_{j, i}=\left[N_{j}-N_{i}, E_{j}-E_{i}\right]^{T} .
$$




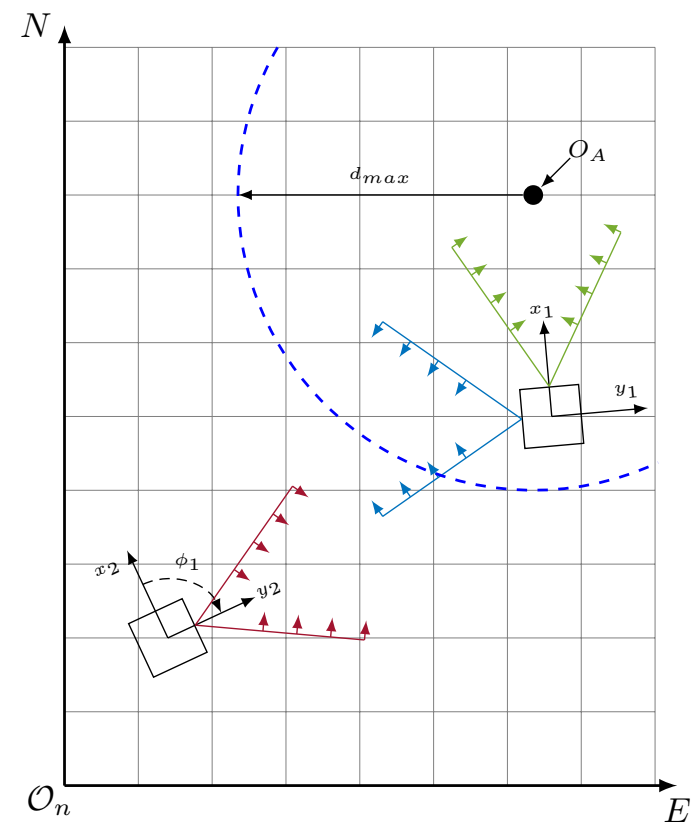

Fig. 1: Figure shows a multi-vehicle system consisting of two vehicles $v_{1}$ and $v_{2}$ as well as an object $O_{A}$. Vehicle $v_{1}$ is equipped with two cameras shown in blue and green, while vehicle $v_{2}$ is equipped with one camera marked with brown. The arrows on the cone-limits mark the direction of the normal vector of the constraint. $\phi_{1}$ on $v_{2}$ shows the relative rotation between the onboard camera and the vehicle.

The parameterizations of the normal vectors $\boldsymbol{n}_{i, j}^{+}$and $\boldsymbol{n}_{i, j}^{-}$ are:

$$
\begin{aligned}
& \boldsymbol{n}_{i, j}^{+}:\left[\begin{array}{ll}
-\sin \left(\xi_{i, k}+\alpha_{k}\right) & \cos \left(\xi_{i, k}+\alpha_{k}\right)
\end{array}\right], \\
& \boldsymbol{n}_{i, j}^{-}:\left[\sin \left(\xi_{i, k}-\alpha_{k}\right)-\cos \left(\xi_{i, k}-\alpha_{k}\right)\right],
\end{aligned}
$$

where $\alpha_{k}$ is the camera half-angle of the visual cone for camera $k, \xi_{i, k}$ is the direction of the cone relative to the global frame $\xi_{i, k}=\psi_{i}+\phi_{k}$ with $\phi_{k}$ being the relative rotation between the vehicle $i$ 's local $x$-axis and the visual cone of camera $k$. Fig. 1 shows the camera cone of two vehicles with respect to each other and a target.

\section{Consensus Problem}

The control objective is to solve the rendezvous problem, meaning the vehicles should to converge to and remain at a common point.

$$
\begin{aligned}
& \lim _{t \rightarrow \infty}\left\|\boldsymbol{\eta}_{i}-\boldsymbol{\eta}_{j}\right\|=0 \forall v_{i} \in \mathcal{V} \wedge v_{j} \in \mathcal{N}_{i}, \\
& \lim _{t \rightarrow \infty}\left\|\boldsymbol{\nu}_{i}\right\|=0 \forall v_{i} \in \mathcal{V} .
\end{aligned}
$$

The rendezvous objective of Eq. (14) is not feasible in practice since the vehicles cannot occupy the same space, however, in combination with the visual constraint the system will converge from a direction defined by the camera cones. Thus the vehicles will collide, but this is the desire as docking requires physical contact. Introducing the vectors $\boldsymbol{x}, \boldsymbol{\eta}, \boldsymbol{\nu}$ and $\boldsymbol{u}$ as follows:

$$
\begin{aligned}
\boldsymbol{\eta} & =\left[\boldsymbol{\eta}_{1}^{T}, \ldots, \boldsymbol{\eta}_{n}^{T}\right]^{T}, \boldsymbol{u}=\left[\boldsymbol{u}_{1}^{T}, \ldots, \boldsymbol{u}_{n}^{T}\right]^{T}, \\
\boldsymbol{\nu} & =\left[\boldsymbol{\nu}_{1}^{T}, \ldots, \boldsymbol{\nu}_{n}^{T}\right]^{T}, \boldsymbol{x}=\left[\boldsymbol{x}_{1}^{T}, \ldots, \boldsymbol{x}_{n}^{T}\right]^{T},
\end{aligned}
$$

where the subscript $\boldsymbol{\eta}_{i}$ denotes the pose of vehicle $v_{i}$. The objective function $J$ of the centralized problem is formulated as follows,

$$
J(\boldsymbol{X}, \boldsymbol{U})=\sum_{k=0}^{N-1} l\left(\boldsymbol{x}_{t+k \mid t}, \boldsymbol{u}_{t+k}\right),
$$

where $\boldsymbol{U}$ is all control inputs for all vehicles across the time horizon $\boldsymbol{U}=\left[\boldsymbol{u}_{t}, \boldsymbol{u}_{t+1}, \ldots, \boldsymbol{u}_{t+N}\right], N$ is the control horizon, $\boldsymbol{X}$ is all the state-vectors over the horizon, and the function $l(\boldsymbol{x}, \boldsymbol{u})$ is defined as:

$$
l(\boldsymbol{x}, \boldsymbol{u})=\boldsymbol{\eta}^{T}(\boldsymbol{L} \otimes \boldsymbol{P}) \boldsymbol{\eta}+\boldsymbol{\nu}^{T} \boldsymbol{W} \boldsymbol{\nu}+\boldsymbol{u}^{T} \boldsymbol{R} \boldsymbol{u},
$$

where matrix $\boldsymbol{L}$ is the Laplacian matrix of the graph, $\otimes$ is the Kronecker product, $\boldsymbol{P}, \boldsymbol{W}$ and $\boldsymbol{R}$ are tuning matrices.

\section{Centralized Problem}

The centralized control problem is composed of the consensus problem and the LOS constraints. The overall controller is formulated as follows:

$$
\begin{aligned}
& \underset{\boldsymbol{U}}{\operatorname{minimize}} J(\boldsymbol{X}, \boldsymbol{U}) \\
& \text { s.t. } \\
& \boldsymbol{u}_{i, t+k} \in \mathcal{U}_{i}, \forall v_{i} \in \mathcal{V}, \\
& \boldsymbol{x}_{i, t+k+1 \mid t}=\boldsymbol{f}_{i}\left(\boldsymbol{x}_{i, t+k \mid t}, \boldsymbol{u}_{i, t+k}\right), \forall v_{i} \in \mathcal{V}, \\
& \boldsymbol{n}_{i, j}^{ \pm} \boldsymbol{e}_{j, i} \leq 0, \forall v_{i} \in \mathcal{V} \wedge v_{j} \in \mathcal{N}_{i}, \\
& \boldsymbol{x}_{t+k \mid t}=\left[\boldsymbol{x}_{1, t+k \mid t}, \boldsymbol{x}_{2, t+k \mid t}, \ldots, \boldsymbol{x}_{n, t+k \mid t}\right], \\
& \boldsymbol{u}_{t+k}=\left[\boldsymbol{u}_{1, t+k}, \boldsymbol{u}_{2, t+k}, \ldots, \boldsymbol{u}_{n, t+k}\right],
\end{aligned}
$$

where $\mathcal{U}_{i}$ is the input constraints for vehicle $v_{i}$

\section{Cooperative Model Predictive Control}

The controller must be converted into a distributed formulation to effectively employ it in a multi-vehicle system. This section describes the usage of dual decomposition to distribute the problem among the agents.

\section{A. Rendezvous Problem}

The rendezvous problem from Sec. III-C is solved purely through the objective function in Eq. (18). However, the function itself relies on the difference in the pose of the vehicles due to $\boldsymbol{L}$. Hence the objective function is not separable. Introducing a new consensus parameter vector $\boldsymbol{\theta} \in \mathbb{R}^{3}$, which acts as a universal rendezvous point for the system, allows for the reformulation of the objective function. The global rendezvous point $\boldsymbol{\theta}$ is then distributed by introducing a local version $\boldsymbol{\theta}_{i}$ on each vehicle and adding the equality constraints as follows,

$$
\boldsymbol{\theta}_{i}=\boldsymbol{\theta}_{j} \forall v_{i} \in \mathcal{V} \wedge v_{j} \in \mathcal{N}_{i} .
$$


The stage cost function of Eq. 17 is reformulated into the following expression:

$l_{i}\left(\boldsymbol{x}_{i}, \boldsymbol{u}_{i}, \boldsymbol{\theta}_{i}\right)=\left(\boldsymbol{\eta}_{i}-\boldsymbol{\theta}_{i}\right)^{T} \boldsymbol{P}\left(\boldsymbol{\eta}_{i}-\boldsymbol{\theta}_{i}\right)+\boldsymbol{\nu}_{i}^{T} \boldsymbol{W} \boldsymbol{\nu}_{i}+\boldsymbol{u}_{i}^{T} \boldsymbol{R} \boldsymbol{u}_{i}$.

The Hessian of $l_{i}$ is only positive-semidefinite, and thus the problem is under-specified. There is no guarantee that the consensus point reaches a steady point unless additional pose constraints are applied, such as a stationary target or target area. The equality constraint of Eq. (20) is subsequently softened using Lagrangian relaxation and added to the objective function in the following section.

\section{B. LOS-Constraint}

The LOS constraints of Eq. (12) depends on the position error $\boldsymbol{e}_{j, i}$ and the normal vectors are parameterized in the attitude of the local vehicle.

$$
\begin{aligned}
& \boldsymbol{n}_{i, j}^{+} \boldsymbol{p}_{j}-\boldsymbol{n}_{i, j}^{+} \boldsymbol{p}_{i} \leq 0 \\
& \boldsymbol{n}_{i, j}^{-\boldsymbol{p}_{j}}-\boldsymbol{n}_{i, j}^{-} \boldsymbol{p}_{i} \leq 0
\end{aligned}
$$

Together, the constraints can be reformulated on matrix form as follows,

$$
\boldsymbol{A}_{i, j} \boldsymbol{x}_{j}-\boldsymbol{A}_{i, j} \boldsymbol{x}_{i} \leq 0 .
$$

Finally, since the constraint is now linear in the state, a local optimization problem can be formulated,

$$
\begin{aligned}
& J_{i}\left(\boldsymbol{X}_{i}, \boldsymbol{U}_{i}, \boldsymbol{\Theta}_{i}\right)=\sum_{k=0}^{N-1}\left[l\left(\boldsymbol{x}_{i, t+k \mid t}, \boldsymbol{u}_{i, t+k}\right)\right. \\
+ & \sum_{j \in \mathcal{N}_{i}}\left[\boldsymbol{\lambda}_{j, i t+k}^{T} \boldsymbol{A}_{j, i}-\boldsymbol{\lambda}_{i, j, t+k}^{T} \boldsymbol{A}_{i, j}\right] \boldsymbol{x}_{i, t+k \mid t} \\
+ & \left.\sum_{j \in \mathcal{N}_{i}^{+}} \boldsymbol{\mu}_{i j, t+k}^{T} \boldsymbol{\theta}_{i, t+k}-\sum_{j \in \mathcal{N}_{i}^{-}} \boldsymbol{\mu}_{i j, t+k}^{T} \boldsymbol{\theta}_{i, t+k}\right],
\end{aligned}
$$

where $\boldsymbol{\lambda}_{j, i}$, and $\boldsymbol{\mu}_{i j}$ are the Lagrangian multipliers associated with the LOS constraint from vehicle $j$ to vehicle $i$ and the consensus equality constraint associated with the edge $e_{i j}$ respectively. $\boldsymbol{X}_{i}$ is the states of vehicle $i$ over the time horizon, $\boldsymbol{X}_{i}=\left[\boldsymbol{x}_{i, t \mid t}, \boldsymbol{x}_{i, t+1 \mid t}, \ldots, \boldsymbol{x}_{i, t+N-1 \mid t}\right], \boldsymbol{U}_{i}$ is the inputs over the time horizon $\boldsymbol{U}_{i}=\left[\boldsymbol{u}_{i, t}, \boldsymbol{u}_{i, t+1}, \ldots, \boldsymbol{u}_{i, t+N-1}\right]$ and $\boldsymbol{\Theta}_{i}$ is the locally computed consensus point across the time horizon, $\boldsymbol{\Theta}_{i}=\left[\boldsymbol{\theta}_{i, t}, \boldsymbol{\theta}_{i, t+1}, \ldots, \boldsymbol{\theta}_{i, t+N-1}\right]$, The control problem can now be reformulated as a local optimization problem as follows:

$$
\begin{aligned}
& \underset{\boldsymbol{X}_{i}, \boldsymbol{U}_{i}, \boldsymbol{\Theta}_{i}}{\operatorname{minimize}} J_{i}\left(\boldsymbol{X}_{i}, \boldsymbol{U}_{i}, \boldsymbol{\Theta}_{i}\right) \\
& \text { s.t. } \\
& \boldsymbol{u}_{i, t+k} \in \mathcal{U}_{i}, \\
& \boldsymbol{x}_{i, t+k+1 \mid t}=\boldsymbol{f}_{i}\left(\boldsymbol{x}_{i, t+k \mid t}, \boldsymbol{u}_{i, t+k}\right),
\end{aligned}
$$

The dual variables $\boldsymbol{\lambda}$ for the visual inequalities are updated using the projected sub-gradient method [3] as follows:

$$
\boldsymbol{\lambda}_{i, j, t+k}^{r+1}=\boldsymbol{\lambda}_{i, j, t+k}^{r}+\kappa_{i, j}^{r}\left(\boldsymbol{A}_{i, j, t+k}\left[\boldsymbol{x}_{j, t+k \mid t}^{* r}-\boldsymbol{x}_{i, t+k \mid t}^{* r}\right]\right)_{+},
$$

where $(\cdot)^{*}$ are the resulting optimal values from the solution to Eq. $(25-26),(\cdot)^{r}$ denotes the $r$ th iteration, $(\cdot)_{+}$is the projected gradient, and $\boldsymbol{A}_{i, j, t+k}$ is the visual constraint matrix updated over the time horizon.

The dual variables $\boldsymbol{\mu}$ for the consensus equality constraints are likewise updated using the sub-gradient method [3].

$$
\boldsymbol{\mu}_{i j, t+k}^{r+1}=\boldsymbol{\mu}_{i j, t+k}^{r}+\gamma_{i j}^{r}\left(\boldsymbol{\theta}_{i, t+k}^{* r}-\boldsymbol{\theta}_{j, t+k}^{* r}\right) .
$$

The overall control algorithm is shown in Algorithm 1.

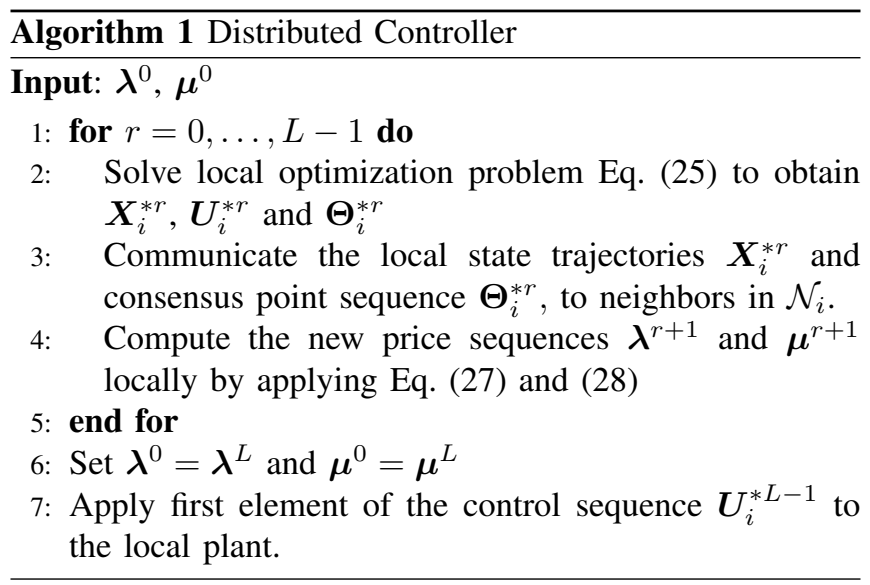

\section{Simulations}

In this section a simulation is conducted to show the application of the controller. The simulation is implemented in Python using CasADI [1]. The model of Eq. (7) is discretized using Runge-Kutta4. The applied optimization solver is the interior-point implementation IPOPT [34].

\begin{tabular}{|ccc|}
\hline Parameter & Value & Unit \\
\hline$\kappa_{i, j}$ & 30 & {$[-]$} \\
\hline$\gamma_{i j}$ & 2 & {$[-]$} \\
\hline $\boldsymbol{D}_{i}$ & $\operatorname{diag}(30,30,1)$ & {$[-]$} \\
\hline $\boldsymbol{M}_{i}$ & $\operatorname{diag}(10,10,1)$ & {$[-]$} \\
\hline$\|\boldsymbol{u}\|_{\infty}$ & {$[5,5,1.5]$} & {$[-]$} \\
\hline$L$ & 15 & {$[-]$} \\
\hline $\boldsymbol{x}_{1,0}$ & {$[4,3,-0.2,0,0,0]$} & {$[-]$} \\
\hline $\boldsymbol{x}_{2,0}$ & {$[1,-2,-0.58,0,0,0]$} & {$[-]$} \\
\hline$O_{A}$ & {$[6.1,3]$} & {$[m]$} \\
\hline$d_{m a x}$ & 3 & {$[m]$} \\
\hline $\boldsymbol{P}_{i}$ & $\operatorname{diag}(15,15,15)$ & {$[-]$} \\
\hline $\boldsymbol{W}_{i}$ & $\operatorname{diag}(10,10,10)$ & {$[-]$} \\
\hline $\boldsymbol{R}_{i}$ & $\operatorname{diag}(10,10,10)$ & {$[-]$} \\
\hline$N$ & 30 & {$[-]$} \\
\hline$\alpha_{k}$ & 25 & {$[$ deg } \\
\hline$\delta t$ & 0.05 & {$[s]$} \\
\hline
\end{tabular}

TABLE I: Parameters and initial conditions used in the simulation scenario.

\section{A. Monitoring and Rendezvous}

The simulation scenario consists of two vehicles $v_{1}$, and $v_{2}$ and a stationary target $O_{A}$. Vehicle $v_{1}$ is inspecting target $O_{A}$, while at the same time rendezvous and docking with vehicle $v_{2}$. The inspecting vehicle is required to stay within 
Camera Bearing Angle between designated targets

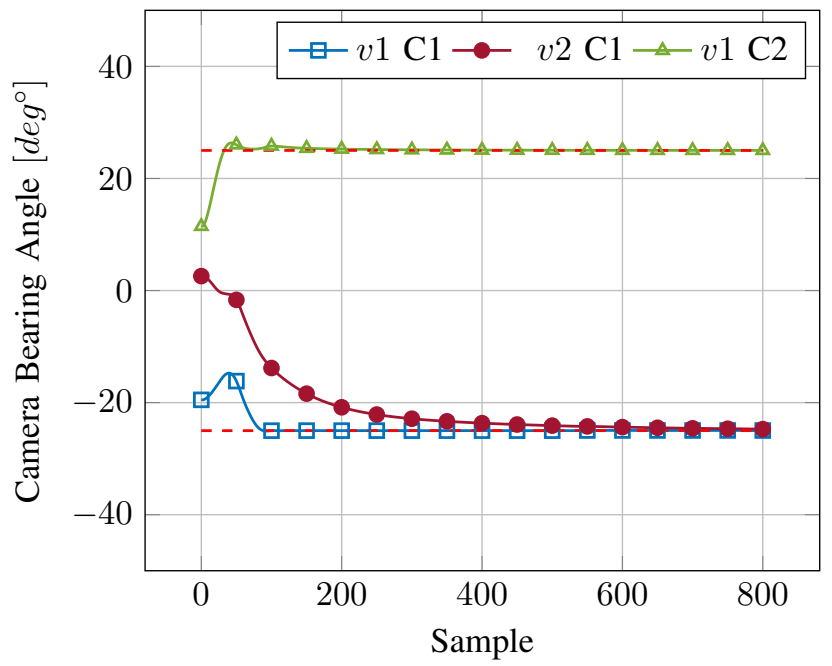

Fig. 2: The figure shows bearing angles in each of the cameras. The bearings of the cameras overshoots the limits slightly and thereby violates the constraint, however, in practice this could be avoided by defining a safety limit a couple of degrees inside the actual fieldof-view

a certain distance of the target and maintain visual contact. Fig. 1 shows the scenario, where $d_{\max }$ is the maximum distance allowed between $v_{1}$ and $O_{A}$. The distance constraint is implemented as a quadratic constraint as follows:

$$
\left(\boldsymbol{p}_{O_{A}}-\boldsymbol{p}_{1}\right)^{T}\left(\boldsymbol{p}_{O_{A}}-\boldsymbol{p}_{1}\right) \leq d_{\max }^{2}
$$

The location of the object $\boldsymbol{p}_{O_{A}}$ is stationary, and known. Thereby, the constraint (29) is simply an inequality constraint on vehicle one. Moreover, the visual constraint (13) is only from the vehicle to the object and requires no dual decomposition and communication between them.

Table I contains the dynamics parameters, initial conditions, and tuning parameters. Fig. 2 shows the bearing angles measured in the cameras. The bearings move towards the visual constraint limits and overshoots slightly. The overshoot is natural since the visual constraints are implemented using Lagrangian relaxation, and the number of iterations for updating the dual variables are limited. The overshoot is easily dealt with if one defines the limits lower than the actual camera field of view. Fig. 3 shows the trajectories of the vehicles. Vehicle $v_{1}$ shown in blue starts inside the designated target area looking at object $O_{A}$, while vehicle $v_{2}$ starts outside the area with $v_{1}$ inside the field-of-view. Note that the constraint of (29) imposes an implicit restriction on the pose of vehicle one. Hence the consensus point of (21) is attracted to the position of vehicle one. The constraint violation is a trade-off between both the tuning of the consensus and the number of iterations for the update of the dual variables and as such more iterations would allow a better estimate of the actual dual variables. The subgradient method is converging

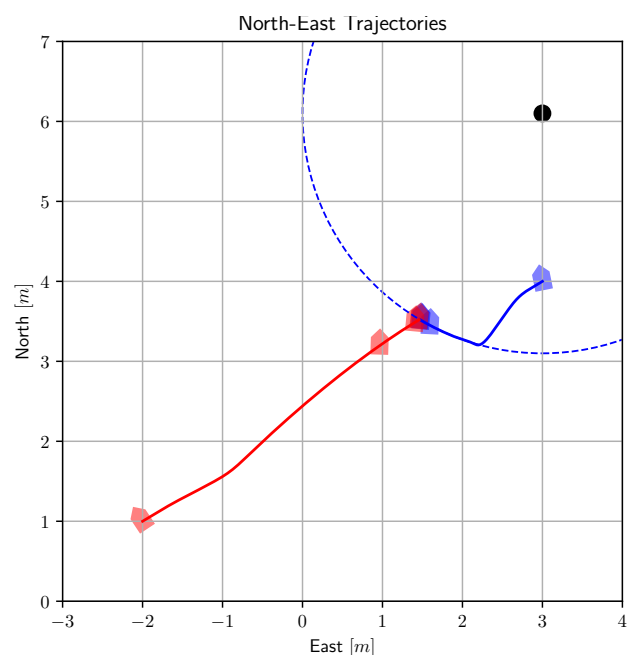

Fig. 3: The trajectories show that each vehicle move towards each other while trying to maintain visual contact. Vehicle $v_{1}$ stays within the prescribed distance of the target while keeping both $v_{1}$ and $O_{A}$ inside the respective camera field-of-view.

at a sub-linear rate and thus to obtain accurate dual variables a large number of iterations are required [13], [15]. The communication requirement quickly increases as a result of the sharing of state trajectories and optimal consensus point sequences. One possible solution would be to allow second-order information to the update of the dual variables at the expense of slightly more data communication for each iteration. The second-order information would allow up to quadratic convergence of the dual variables and as such reduce the necessary iterations significantly [13], [15]. The bandwidth of underwater optical communication would allow for the increased communication requirement within a few tens of meters [36], [35]. The communication requirement for the specific simulation was approximately $1.7 \mathrm{Mbit} / \mathrm{s}$. The bandwidth of acoustic communication range up to 0.5 Mbit/s at $60 \mathrm{~m} \mathrm{[30],} \mathrm{but} \mathrm{the} \mathrm{bandwidth} \mathrm{can} \mathrm{by} \mathrm{manipulation}$ of the sampling time $\delta t$, the max iteration $L$ and the length of the control horizon $N$ be significantly lowered.

\section{CONCLUSION}

In this paper, we presented a distributed model predictive controller for the solution to a cooperative rendezvous and docking problem under visual camera constraints. The problem consisted of two subparts, namely a consensus problem and a visual field-of-view restriction. A DMPC was derived using dual decomposition. A simulation study was conducted to show the application of the controller, where two vehicles conduct are rendezvous and docking maneuver while one of the vehicles stay within a maximum prescribed distance of a designated target and maintain visual contact.

The present communication technology does allow the proposed method to work, but for robustness reduction in communication is a topic of future research. 


\section{REFERENCES}

[1] J. Andersson. A General-Purpose Software Framework for Dynamic Optimization. Phd, Arenberg Doctoral School, KU Leuven.

[2] P. Batista, C. Silvestre, and P. Oliveira. A two-step control approach for docking of autonomous underwater vehicles. International Journal of Robust and Nonlinear Control, 25(10):1528-1547, jul 2015.

[3] D. P. Bertsekas. Nonlinear programming. Athena Scientific, 2nd edition, Sept. 2008.

[4] R. Capocci, G. Dooly, E. Omerdić, J. Coleman, T. Newe, and D. Toal. Inspection-Class Remotely Operated VehiclesâĂŤA Review. Journal of Marine Science and Engineering, 5(1):13, 2017.

[5] Z. Cheng, H.-T. Zhang, and M.-C. Fan. Consensus and rendezvous predictive control for multi-agent systems with input constraints. In Proceedings of the 33rd Chinese Control Conference, pages 14381443. IEEE, jul 2014.

[6] B. N. Circle. Underwater mobile docking of autonomous underwater vehicles. In 2012 Oceans, pages 1-15. IEEE, oct 2012.

[7] R. Conti, E. Meli, a. Ridolfi, and B. Allotta. An innovative decentralized strategy for I-AUVs cooperative manipulation tasks. Robotics and Autonomous Systems, 72:261-276, 2015.

[8] G. Ferrari-Trecate, L. Galbusera, M. Marciandi, and R. Scattolini. Model Predictive Control Schemes for Consensus in Multi-Agent Systems with Single- and Double-Integrator Dynamics. IEEE Transactions on Automatic Control, 54(11):2560-2572, nov 2009.

[9] P. Giselsson and A. Rantzer. Distributed Model Predictive Control with suboptimality and stability guarantees. In 49th IEEE Conference on Decision and Control (CDC), pages 7272-7277. IEEE, dec 2010.

[10] A. Grancharova and T. A. Johansen. Distributed quasi-nonlinear model predictive control by dual decomposition. In Proceedings of the 18th World Congress, volume 18, pages 1429-1434. IFAC, 2011.

[11] E. H. Henriksen, T. Berge Gjersvik, and B. Thorkildsen. Positioning of subsea modules using an automated ROV. In OCEANS 2015 Genova, pages 1-8. IEEE, may 2015.

[12] T. Keviczky and K. H. Johansson. A Study On Distributed Model Predictive Consensus. In Proceedings of the 17th World Congress, volume 41, pages 1516-1521. IFAC, 2008.

[13] E. Klintberg and S. Gros. A primal-dual Newton method for distributed Quadratic Programming. In 53rd IEEE Conference on Decision and Control, pages 5843-5848. IEEE, dec 2014.

[14] A. Kozma, J. V. Frasch, and M. Diehl. A distributed method for convex quadratic programming problems arising in optimal control of distributed systems. In 52nd IEEE Conference on Decision and Control, pages 1526-1531. IEEE, dec 2013.

[15] A. Kozma, E. Klintberg, S. Gros, and M. Diehl. An improved distributed dual newton-CG method for convex quadratic programming problems. Proceedings of the American Control Conference, pages 2324-2329, 2014.

[16] D. M. Lane, F. Maurelli, P. Kormushev, M. Carreras, M. Fox, and K. Kyriakopoulos. Persistent autonomy: the challenges of the PANDORA project. In 9th IFAC Conference on Manoeuvring and Control of Marine Craft, volume 45, pages 268-273. IFAC, 2012.

[17] P. Li and Z. H. Zhu. Line-of-sight nonlinear model predictive control for autonomous rendezvous in elliptical orbit. Aerospace Science and Technology, 69:236-243, 2017.

[18] Q. Li, J. Yuan, B. Zhang, and C. Gao. Model predictive control for autonomous rendezvous and docking with a tumbling target. Aerospace Science and Technology, 1:1-12, 2017.

[19] S. Maniatopoulos, D. Panagou, and K. J. Kyriakopoulos. Model Predictive Control for the navigation of a nonholonomic vehicle with field-of-view constraints. American Control Conference (ACC), 2013, pages 3967-3972, 2013.
[20] F. Maurelli, M. Carreras, J. Salvi, D. Lane, K. Kyriakopoulos, G. Karras, M. Fox, D. Long, P. Kormushev, and D. Caldwell. The PANDORA project: A success story in AUV autonomy. OCEANS 2016 - Shanghai, 2016.

[21] N. Palomeras, A. Carrera, N. Hurtós, G. C. Karras, C. P. Bechlioulis, M. Cashmore, D. Magazzeni, D. Long, M. Fox, K. J. Kyriakopoulos, P. Kormushev, J. Salvi, and M. Carreras. Toward persistent autonomous intervention in a subsea panel. Autonomous Robots, 40(7):1279-1306, 2016.

[22] N. Palomeras, A. Peñalver, M. Massot-Campos, P. Negre, J. Fernández, P. Ridao, P. Sanz, and G. Oliver-Codina. I-AUV Docking and Panel Intervention at Sea. Sensors, 16(10):1673, oct 2016.

[23] H. Park, S. D. Cairano, and I. Kolmanovsky. Model Predictive Control for Spacecraft Rendezvous and Docking with a Rotating / Tumbling Platform and for Debris Avoidance. American Control Conference, pages 1922-1927, 2011.

[24] H. Park, S. Di Cairano, and I. Kolmanovsky. Model Predictive Control of spacecraft docking with a non-rotating platform. In Proceedings of the 18th World Congress, volume 18, pages 8485-8490. IFAC, 2011.

[25] H. Park, C. Zagaris, J. Virgili Llop, R. Zappulla, I. Kolmanovsky, and M. Romano. Analysis and Experimentation of Model Predictive Control for Spacecraft Rendezvous and Proximity Operations with Multiple Obstacle Avoidance. AIAA/AAS Astrodynamics Specialist Conference, (September):1-17, 2016.

[26] A. Rantzer. Dynamic dual decomposition for distributed control. Proceedings of the American Control Conference, pages 884-888, 2009.

[27] P. Sanz, M. Prats, P. Ridao, D. Ribas, G. Oliver, and A. Ortiz. Recent progress in the RAUVI project: A Reconfigurable autonomous underwater vehicle for intervention. In ELMAR, 2010 Proceedings, number September, pages 471-474. IEEE, 2010.

[28] I. Schjølberg, T. B. Gjersvik, A. A. Transeth, and I. B. Utne. Next Generation Subsea Inspection, Maintenance and Repair Operations. In 10th IFAC Conference on Control Applications in Marine Systems (CAMS 2016), volume 49, pages 434-439. Elsevier B.V., 2016.

[29] I. Schjølberg and I. B. Utne. Towards autonomy in ROV operation. 4th IFAC Workshop on Navigation, Guidance and Control of Underwater Vehicles (NGCUV), pages 183-188, 2015.

[30] M. Stojanovic and P.-P. J. Beaujean. Acoustic Communication. Springer Handbook of Ocean Engineering, pages 359-386, 2016.

[31] C. A. Thieme and I. B. Utne. A risk model for autonomous marine systems and operation focusing on humanâĂŞautonomy collaboration. Proceedings of the Institution of Mechanical Engineers, Part $O$ : Journal of Risk and Reliability, 231(4):446-464, 2017.

[32] C. A. Thieme, I. B. Utne, and I. Schjølberg. A Risk Management Framework for Unmanned Underwater Vehicles Focusing on Human and Organizational Factors. In ASME 2015 34th International Conference on Ocean, Offshore and Arctic Engineering, page V003T02A075. ASME, may 2015.

[33] I. Vasilescu, C. Detweiler, M. Doniec, D. Gurdan, S. Sosnowski, J. Stumpf, and D. Rus. AMOUR V: A Hovering Energy Efficient Underwater Robot Capable of Dynamic Payloads. The International Journal of Robotics Research, 29(5):547-570, 2010.

[34] A. Wächter and L. T. Biegler. On the implementation of an interiorpoint filter line-search algorithm for large-scale nonlinear programming. Mathematical Programming, 106(1):25-57, mar 2006.

[35] C. Wang, H. Y. Yu, Y. J. Zhu, T. Wang, and Y. W. Ji. Multi-LED parallel transmission for long distance underwater VLC system with one SPAD receiver. Optics Communications, 410(March 2018):889895, 2018.

[36] Z. Zeng, S. Fu, H. Zhang, Y. Dong, and J. Cheng. A Survey of Underwater Optical Wireless Communications. IEEE Communications Surveys \& Tutorials, 19(1):204-238, 2017. 